TRANSACTIONS OF THE

AMERICAN MATHEMATICAL SOCIETY

Volume 360, Number 10, October 2008, Pages 5101-5120

S 0002-9947(08)04494-2

Article electronically published on May 19, 2008

\title{
BLOW-UP IN FINITE TIME FOR THE DYADIC MODEL OF THE NAVIER-STOKES EQUATIONS
}

\author{
ALEXEY CHESKIDOV
}

\begin{abstract}
We study the dyadic model of the Navier-Stokes equations introduced by Katz and Pavlović. They showed a finite time blow-up in the case where the dissipation degree $\alpha$ is less than $1 / 4$. In this paper we prove the existence of weak solutions for all $\alpha$, energy inequality for every weak solution with nonnegative initial data starting from any time, local regularity for $\alpha>1 / 3$, and global regularity for $\alpha \geq 1 / 2$. In addition, we prove a finite time blow-up in the case where $\alpha<1 / 3$. It is remarkable that the model with $\alpha=1 / 3$ enjoys the same estimates on the nonlinear term as the $4 \mathrm{D}$ NavierStokes equations. Finally, we discuss a weak global attractor, which coincides with a maximal bounded invariant set for all $\alpha$ and becomes a strong global attractor for $\alpha \geq 1 / 2$.
\end{abstract}

\section{INTRODUCTION}

The regularity of the 3D incompressible Navier-Stokes equations (NSE) remains a significant problem. This, among many other open problems connected with the 3D NSE, depends on the estimates on the inertial term $(\mathbf{u} \cdot \nabla) \mathbf{u}$ in the equations. In this paper we study a dyadic model, which has similar properties to the 3D NSE, the same estimates on the inertial term, and the same open question concerning the regularity of the solutions.

There have been many simple models proposed in the literature that capture some essential features of the 3D NSE. Among these are shell models of turbulence, which have been investigated for many years (see [2, 11, 12, 16, 18]). Recently, some of these models, as well as some new ones, were extensively studied analytically. In [7, Constantin, Levant, and Titi study the "sabra" shell model of turbulence, proving a global regularity and the existence of a finite dimensional global attractor and inertial manifold.

In 14, Katz and Pavlović introduced another shell-type model, the dyadic model for the Euler and Navier-Stokes equations. This model, motivated by [13, is an infinite system of nonlinear ODEs that describes evolutions of wavelet coefficients. In [9, Friedlander and Pavlović proposed a three-dimensional vector model for the Euler equations, similar to a quasi-linear approximation of the 3D Navier-Stokes system constructed by Dinaburg and Sinai 8 . Both of these dyadic models can be reduced to the following system of nonlinear ODEs:

$$
\frac{d}{d t} u_{n}+\nu \lambda^{2 \alpha n} u_{n}-\lambda^{n} u_{n-1}^{2}+\lambda^{n+1} u_{n} u_{n+1}=g_{n}, \quad n \in \mathbb{N},
$$

Received by the editors January 4, 2006.

2000 Mathematics Subject Classification. Primary 35Q30, 76D03, 76D05.

(C)2008 American Mathematical Society 
where $u_{0}=0$. Here, $\lambda>1, \nu \geq 0$ is the viscosity, and $\alpha>0$ is the dissipation degree. Note that we also include a force $g=\left(g_{1}, g_{2}, \ldots\right)$ in the model. For $u=\left(u_{1}, u_{2}, \ldots\right)$, the dyadic model can be written as

$$
\frac{d}{d t} u+\nu A u+B(u, u)=g
$$

where

$$
(A u)_{n}=\lambda^{2 \alpha n} u_{n}, \quad(B(u, u))_{n}=-\lambda^{n} u_{n-1}^{2}+\lambda^{n+1} u_{n} u_{n+1},
$$

and $u_{0}=0$. Note that for $\alpha=2 / 5$ the following are sharp estimates on the inertial term:

$$
|(B(u, u), A u)| \lesssim|A u|^{3 / 2}\left|A^{1 / 2} u\right|^{3 / 2},
$$

where $(\cdot, \cdot)$ and $|\cdot|$ are the $l^{2}$-inner product and norm respectively. The best known estimates on the inertial term of the 3D NSE are the same, with $(\cdot, \cdot)$ and $|\cdot|$ being the $L^{2}$-inner product and norm.

Katz and Pavlović [14] proved that under certain assumptions on the initial conditions, solutions of the inviscid dyadic model blow up in finite time in a norm stronger than the $l^{2}$-norm. Later, Waleffe [21] derived the inviscid dyadic model from the Burgers equation reducing it to (1.1) with $\nu=0$. Recently, Kiselev and Zlatoš 15 sharpened the blow-up result for the dyadic model and studied a very similar Obukhov model (see [17]) proving a global regularity of every solution with regular initial data. In addition, the existence of a global attractor of the inviscid dyadic model is proved in [5]. This surprising fact is a result of a self-dissipation mechanism due to the loss of regularity of solutions.

In this paper we will study the viscous dyadic model, i.e., the model (1.1) with $\nu>0$. In this case Katz and Pavlović [14] obtained a finite time blow-up of solutions with certain initial data when $\alpha<1 / 4$. Our main goal is to prove a finite time blow-up in the case where $\alpha<1 / 3$. It is remarkable that in the critical case $\alpha=1 / 3$ the following are sharp estimates on the nonlinear term:

$$
|(B(u, u), A u)| \lesssim|A u|^{2}\left|A^{1 / 2} u\right| .
$$

Note that the best known estimates on the nonlinear term of the 4D NSE are exactly the same.

This paper is structured as follows. We start with surveying the dyadic model in Section 2, In Section 3 we introduce a functional setting and define weak and strong solutions. In Section 4 we derive some a priori estimates and prove the existence of weak solutions to the dyadic model. This is done by taking a limit of the Galerkin approximation, which also results in the energy inequality for a limit solution (which might not be unique) for almost all time. Then we show that the lack of backward energy transfer implies that every weak solution with nonnegative initial data satisfies the energy inequality starting from any time. Finally, using a classical NSE technique, we show a local regularity for $\alpha>1 / 3$ and a global regularity for $\alpha \geq 1 / 2$.

In Section 5, inverting the Sobolev-type estimates, we prove that every solution with large $H^{\epsilon}$-norm blows up in finite time in the $H^{1 / 3+\epsilon}$-norm, $\epsilon>0$. Here, the $H^{\gamma}$-norm is defined as $\|u\|_{\gamma}=\left(\sum \lambda^{2 \gamma n} u_{n}^{2}\right)^{1 / 2}$. Note that we also use such a technique in 4], where we study a similar model that has coefficients growing as power functions. That model is introduced as an example of an NSE-like dynamical system that possesses a weak global attractor, on which all the solutions blow up in finite time. The reason for the power-law growth for the coefficients is to mimic 
the growth of eigenvalues of the Stokes operator in 3D. It is remarkable that due to a slower growth of the coefficients, the model in [4] still possesses a gap between the regions of a local regularity and finite time blow-up.

Lastly, in Section 6 we discuss a weak global attractor for the dyadic model. The weak global attractor is the minimal weakly closed weakly attracting set. Using results from [3, 4, we show that the weak global attractor is also the maximal bounded invariant set. Moreover, for $\alpha \geq 1 / 2$ all the trajectories are continuous in $l^{2}$, which implies that the weak global attractor is in fact a strongly compact strong global attractor.

Note that there is still a gap between the regions of global regularity and blow-up in finite time, which means that the developed technique is not sharp enough to separate these two behaviors. Since most of the proofs in the theory of the NavierStokes equations go through for the dyadic model, a better understanding of the dyadic or similar shell models might provide insight into the regularity problem for the Navier-Stokes equations.

\section{DyADIC MODEL}

Here we recall a derivation of the dyadic model for the equations of fluid motion by Katz and Pavlović 14 . A cube $Q \subset \mathbb{R}^{3}$ is called dyadic if its side length is $2^{l}$, and the corners are on the lattice $2^{l} \mathbb{Z}^{3}$, for some integer $l$. For a dyadic cube $Q$ with side length $2^{-j}$, its parent $\tilde{Q}$ is a unique dyadic cube with side length $2^{-j+1}$ that contains $Q$. For $m \geq 1$, let $C^{m}(Q)$ be the set of all $m$ th order grandchildren of $Q$, i.e., all the dyadic cubes with side length $2^{-j-m}$ that are contained in $Q$. For instance, $C^{1}(Q)$ consists of $2^{3}$ children of $Q$.

Now a scalar-valued function $u(x, t)$ can be represented by the following wavelet expansion:

$$
u(x, t)=\sum_{Q} u_{Q}(t) w_{Q}(x),
$$

where $\left\{w_{Q}\right\}$ is an orthonormal in $L^{2}(\mathbb{R})$ family of wavelets, such that $w_{Q}$ is localized on $Q$. Define the Laplacian in the following way:

$$
\Delta u=\sum_{Q} 2^{2 j(Q)} u_{Q}(t) \omega_{Q}(x)
$$

where $2^{-j(Q)}$ is a side length of a dyadic cube $Q$. Katz and Pavlović define the cascade operator as follows:

$$
(C(u, v))_{Q}=-2^{\frac{5 j}{2}} u_{\tilde{Q}} v_{\tilde{Q}}+2^{\frac{5(j+1)}{2}} u_{Q} \sum_{Q^{\prime} \in C^{1}(Q)} v_{Q^{\prime}}
$$

where $Q$ is a dyadic cube with side length $2^{-j}$. The dyadic Navier-Stokes equation with hypo-dissipation is written as

$$
\frac{d}{d t} u+C(u, u)+\nu(\Delta)^{\alpha} u=0
$$

where we include the viscosity $\nu$, which is chosen to be one in [14. In terms of the wavelet coefficients $u_{Q}$, this equation can be written as

$$
\frac{d}{d t} u_{Q}(t)=-\nu 2^{2 \alpha j} u_{Q}(t)+2^{\frac{5 j}{2}} u_{\tilde{Q}}^{2}(t)-2^{\frac{5(j+1)}{2}} u_{Q} \sum_{Q^{\prime} \in C^{1}(Q)} u_{Q^{\prime}}(t)
$$


As was proposed in 21 in the case $\nu=0$, we simplify the model in the following way. Let $Q_{1}$ be a dyadic cube with side length $2^{-1}$. Let $v_{1}(t)=u_{Q_{1}}(t)$ and $v_{j}(t)=u_{Q_{j}}(t)$, where $Q_{j}$ is some dyadic cube in $C^{j-1}\left(Q_{1}\right), j \geq 2$. We will only consider the initial conditions for which $u_{Q}\left(t_{0}\right)=v_{j}\left(t_{0}\right)$ for all cubes $Q \in C^{j-1}\left(Q_{1}\right)$ for $j \geq 2$, and $u_{Q}\left(t_{0}\right)=0$ for all dyadic cubes with side length larger than $2^{-1}$. Then, for every $j \geq 2$, we have $u_{Q}(t)=v_{j}(t)$ for all $Q \in C^{j-1}\left(Q_{1}\right)$. Now, denoting $v_{0}=0$, we obtain the following system of equations for $v_{j}(t)$ :

$$
\frac{d}{d t} v_{j}(t)=-\nu 2^{2 \alpha j} v_{j}(t)+2^{\frac{5 j}{2}} v_{j-1}^{2}-2^{3} 2^{\frac{5(j+1)}{2}} v_{j} v_{j+1}, \quad j \geq 1 .
$$

Finally, the change of variables

$$
u_{j}(t)=2^{\frac{3 j}{2}} v_{j}(t / 8)
$$

reduces the equations to

$$
\frac{d}{d t} u_{j}=-\tilde{\nu} 2^{2 \alpha j} u_{j}+2^{j} u_{j-1}^{2}-2^{j+1} u_{j} u_{j+1}, \quad j \geq 1,
$$

with $\tilde{\nu}=\nu / 8$.

\section{Functional Setting}

Let us denote $H=l^{2}$ with the usual scalar product and norm:

$$
(u, v):=\sum_{n=1}^{\infty} u_{n} v_{n}, \quad|u|:=\sqrt{(u, u)} .
$$

The norm $|u|$ will be called the energy norm. Let $A: D(A) \rightarrow H$ be the Laplace operator defined by

$$
(A u)_{n}=\lambda^{2 \alpha n} u_{n}, \quad n \geq 1,
$$

for some $\lambda>1$. The domain $D(A)$ of this operator is a dense subset of $H$. Note that $A$ is a positive definite operator whose eigenvalues are

$$
0<\lambda^{2 \alpha} \leq \lambda^{4 \alpha} \leq \lambda^{6 \alpha} \leq \ldots
$$

Let $H^{\gamma}=A^{-\gamma /(2 \alpha)} H$ be endowed with the following scalar product and norm:

$$
((u, v))_{\gamma}:=\sum_{n=1}^{\infty} \lambda^{2 \gamma n} u_{n} v_{n}, \quad\|u\|_{\gamma}:=\sqrt{((u, u))_{\gamma}} .
$$

In the special case $\gamma=\alpha$, let $V=H^{\alpha}=A^{-1 / 2} H$ and

$$
((u, v)):=((u, v))_{\alpha}, \quad\|u\|:=\|u\|_{\alpha} .
$$

This double norm $\|u\|$ will be called the enstrophy norm. Note that we have an equivalent of the Poincaré inequality

$$
|u|^{2} \leq \frac{1}{\lambda^{2 \alpha}}\|u\|^{2}
$$

Also, let

$$
\mathrm{d}_{\mathrm{s}}(u, v):=|u-v|, \quad \mathrm{d}_{\mathrm{w}}(u, v):=\sum_{n=1}^{\infty} \frac{1}{2^{\left(n^{2}\right)}} \frac{\left|u_{n}-v_{n}\right|}{1+\left|u_{n}-v_{n}\right|}, \quad u, v \in H .
$$


Here, $d_{\mathrm{s}}$ is a strong distance, and $d_{\mathrm{w}}$ is a weak distance that induces a weak topology on any bounded subset of $H$. Hence, a bounded sequence $\left\{u^{k}\right\} \subset H$ converges to $u \in H$ weakly, i.e.,

$$
\lim _{k \rightarrow \infty}\left(u^{k}, v\right)=(u, v), \quad \forall v \in H
$$

if and only if

$$
\mathrm{d}_{\mathrm{w}}\left(u^{k}, u\right) \rightarrow 0 \quad \text { as } \quad k \rightarrow \infty .
$$

We also recall that if $u^{k} \rightarrow u$ weakly in $H$ as $k \rightarrow \infty$, then

$$
\liminf _{k \rightarrow \infty}\left|u^{k}\right| \geq|u| \text {. }
$$

Let

$$
C\left([0, T] ; H_{\mathrm{w}}\right):=\left\{u(\cdot):[0, T] \rightarrow H, u_{n}(t) \text { is continuous for all } n\right\}
$$

be endowed with the distance

$$
d_{C\left([0, T] ; H_{\mathrm{w}}\right)}(u, v)=\sup _{t \in[0, T]} \mathrm{d}_{\mathrm{w}}(u(t), v(t)) .
$$

Also, let

$$
C\left([0, \infty) ; H_{\mathrm{w}}\right):=\left\{u(\cdot):[0, \infty) \rightarrow H, u_{n}(t) \text { is continuous for all } n\right\}
$$

be endowed with the distance

$$
d_{C\left([0, \infty) ; H_{\mathrm{w}}\right)}(u, v)=\sum_{T \in \mathbb{N}} \frac{1}{2^{T}} \frac{\sup \left\{\mathrm{d}_{\mathrm{w}}(u(t), v(t)): 0 \leq t \leq T\right\}}{1+\sup \left\{\mathrm{d}_{\mathrm{w}}(u(t), v(t)): 0 \leq t \leq T\right\}} .
$$

In this paper, the dyadic model of the Navier-Stokes equations will be written as

$$
\left\{\begin{array}{l}
\frac{d}{d t} u_{n}+\nu \lambda^{2 \alpha n} u_{n}-\lambda^{n} u_{n-1}^{2}+\lambda^{n+1} u_{n} u_{n+1}=g_{n}, \quad n=1,2,3 \ldots \\
u_{0}=0
\end{array}\right.
$$

for some parameter $\lambda>1$, the viscosity $\nu>0$, the dissipation degree $\alpha>0$, and the force $g=\left(g_{1}, g_{2}, \ldots\right)$. For simplicity, assume that $g$ is independent of time, $g \in H$, and $g_{n} \geq 0$ for all $n$.

For $u=\left(u_{1}, u_{2}, \ldots\right)$, the dyadic model can be written in a more condensed form as

$$
\frac{d}{d t} u+\nu A u+B(u, u)=g
$$

where

$$
(B(u, v))_{n}= \begin{cases}-\lambda^{n} u_{n-1} v_{n-1}+\lambda^{n+1} u_{n} v_{n+1}, & n=2,3, \ldots \\ \lambda^{2} u_{1} v_{2}, & n=1 .\end{cases}
$$

Clearly, the bilinear operator $B$ enjoys the orthogonality property:

$$
\begin{aligned}
(B(u, v), v) & =\sum_{n=1}^{\infty}\left(-\lambda^{n} u_{n-1} v_{n-1} v_{n}+\lambda^{n+1} u_{n} v_{n+1} v_{n}\right) \\
& =\sum_{n=1}^{\infty}\left(-\lambda^{n} u_{n-1} v_{n-1} v_{n}+\lambda^{n} u_{n-1} v_{n} v_{n-1}\right) \\
& =0
\end{aligned}
$$

Note that we always use a convention that $u_{0}=0$. 
Definition 3.1. A weak solution on $[T, \infty)($ or $(-\infty, \infty)$, if $T=-\infty)$ of (3.1) is an $H$-valued function $u(t)$ defined for $t \in[T, \infty)$, such that $u_{n} \in C^{1}([T, \infty))$ and $u_{n}(t)$ satisfies (3.1) for all $n$.

Note that since $(B(u, u))_{n}$ has a finite number of terms, the notions of a weak solution and a classical solution (of a system of ODEs) coincide. Hence, the weak solutions will often be called solutions in the remainder of the paper. Note that if $u(t)$ is a solution on $[T, \infty)$, then automatically $u_{n} \in C^{\infty}([T, \infty))$. We say that a solution $u(t)$ is strong (or regular) on some interval $\left[T_{1}, T_{2}\right]$ if $\|u(t)\|$ is bounded on $\left[T_{1}, T_{2}\right]$. A solution is strong on $\left[T_{1}, \infty\right)$ if it is strong on every interval $\left[T_{1}, T_{2}\right]$, $T_{2} \geq T_{1}$.

Definition 3.2. A Leray-Hopf solution of (3.1) on the interval $[T, \infty)$ is a weak solution of (3.1) on $[T, \infty)$ satisfying the energy inequality

$$
|u(t)|^{2}+2 \nu \int_{t_{0}}^{t}\|u(\tau)\|^{2} d \tau \leq\left|u\left(t_{0}\right)\right|^{2}+2 \int_{t_{0}}^{t}(g, u(\tau)) d \tau
$$

for all $T \leq t_{0} \leq t, t_{0}$ a.e. in $[T, \infty)$. The set $E x$ on which the energy inequality does not hold will be called the exceptional set.

Note that the complement of the exceptional set Ex coincides with the set of points of strong continuity from the right. Later we will prove that every solution $u(t)$ with $u_{n}(T) \geq 0$ is a Leray-Hopf solution on $[T, \infty)$, and that the energy inequality for such a solution is satisfied starting from any time $t_{0} \geq T$, i.e., $E x=\emptyset$.

\section{A priori estimates And existence of WeAK AND Strong SOlutions}

We start with some a priori estimates.

Energy estimates. Formally taking a scalar product of the equation (3.1) with $u$, we obtain

$$
\begin{aligned}
\frac{1}{2} \frac{d}{d t}|u|^{2} & \leq-\nu\|u\|^{2}+|g||u| \\
& \leq-\nu|u|^{2}+\frac{\nu}{2}|u|^{2}+\frac{|g|^{2}}{2 \nu} \\
& =-\frac{\nu}{2}|u|^{2}+\frac{|g|^{2}}{2 \nu} .
\end{aligned}
$$

Using Gronwall's inequality, we conclude that

$$
|u(t)|^{2} \leq e^{-\nu t}|u(0)|^{2}+\frac{|g|^{2}}{\nu^{2}}\left(1-e^{-\nu t}\right) .
$$

Hence, $B=\{u \in H:|u| \leq R\}$ is an absorbing ball for the Leray-Hopf solutions, where $R$ is any number larger than $|g| / \nu$. Note that this result will later follow rigorously from the energy inequality.

Next, taking a limit of the Galerkin approximation, we will prove the existence of Leray-Hopf solutions to (3.1).

Theorem 4.1. For every $u^{0} \in H$ and $g \in H$, there exists a solution $u(t)$ of (3.1) with $u(0)=u^{0}$. Moreover, the energy inequality

$$
|u(t)|^{2}+2 \nu \int_{t_{0}}^{t}\|u(\tau)\|^{2} d \tau \leq\left|u\left(t_{0}\right)\right|^{2}+2 \int_{t_{0}}^{t}(g, u(\tau)) d \tau
$$

holds for all $0 \leq t_{0} \leq t, t_{0}$ a.e. in $[0, \infty)$. 
Proof. Let $u^{0} \in H$. We will show the existence of a weak solution by taking a limit of the Galerkin approximation $u^{k}(t)=\left(u_{1}^{k}(t), \ldots, u_{k}^{k}(t), 0,0, \ldots\right)$ with $u_{n}^{k}(0)=u_{n}^{0}$ for $n=1,2, \ldots, k$, which satisfies

$$
\left\{\begin{array}{l}
\frac{d}{d t} u_{n}^{k}+\nu \lambda^{2 \alpha n} u_{n}^{k}-\lambda^{n}\left(u_{n-1}^{k}\right)^{2}+\lambda^{n+1} u_{n}^{k} u_{n+1}^{k}=g_{n}, \quad n \leq k-1, \\
\frac{d}{d t} u_{k}^{k}+\nu \lambda^{2 \alpha k} u_{k}^{k}-\lambda^{k}\left(u_{k-1}^{k}\right)^{2}=g_{k}
\end{array}\right.
$$

where $u_{0}^{k}=0$. First, note that the energy estimate (4.1) obviously holds for $u^{k}(t)$. Hence, from the theory of ordinary differential equations we know that there exists a unique solution $u^{k}(t)$ to (4.2) on $[0, \infty)$. Next, we will show that a sequence of the Galerkin approximations $\left\{u^{k}\right\}$ is weakly equicontinuous. Indeed, thanks to the energy estimate (4.1), there exists $M$ such that

$$
u_{n}^{k}(t) \leq M, \quad \forall n, k, t \geq 0 .
$$

Therefore,

$$
\begin{aligned}
\left|u_{n}^{k}(t)-u_{n}^{k}(s)\right| & \leq\left|\int_{s}^{t}\left(-\nu \lambda^{2 \alpha n} u_{n}^{k}+\lambda^{n}\left(u_{n-1}^{k}\right)^{2}-\lambda^{n+1} u_{n}^{k} u_{n+1}^{k}+g_{n}\right) d \tau\right| \\
& \leq\left(\nu \lambda^{2 \alpha n} M+\lambda^{n} M^{2}+\lambda^{n+1} M^{2}+g_{n}\right)|t-s|,
\end{aligned}
$$

for all $n, k, t \geq 0, s \geq 0$. Thus,

$$
\begin{aligned}
\mathrm{d}_{\mathrm{w}}\left(u^{k}(t), u^{k}(s)\right) & =\sum_{n=1}^{\infty} \frac{1}{2^{\left(n^{2}\right)}} \frac{\left|u_{n}^{k}(t)-u_{n}^{k}(s)\right|}{1+\left|u_{n}^{k}(t)-u_{n}^{k}(s)\right|} \\
& \leq c|t-s|,
\end{aligned}
$$

for some constant $c$ independent of $k$. Hence, $\left\{u^{k}\right\}$ is an equicontinuous sequence of functions in $C\left([0, \infty) ; H_{\mathrm{w}}\right)$ with bounded initial data. Therefore, the AscoliArzela theorem implies that $\left\{u^{k}\right\}$ is relatively compact in $C\left([0, T] ; H_{\mathrm{w}}\right)$ for every $T \geq 0$. By a diagonalization process it follows that $\left\{u^{k}\right\}$ is relatively compact in $C\left([0, \infty) ; H_{\mathrm{w}}\right)$. Hence, passing to a subsequence, we obtain that there exists a weakly continuous $H$-valued function $u(t)$ such that

$$
u^{k_{j}} \rightarrow u \quad \text { as } \quad k_{j} \rightarrow \infty \quad \text { in } \quad C\left([0, \infty) ; H_{\mathrm{w}}\right) .
$$

In particular, $u_{n}^{k_{j}}(t) \rightarrow u_{n}(t)$ as $k_{j} \rightarrow \infty$, for all $n, t \geq 0$. Thus, $u(0)=u^{0}$. In addition, note that

$$
u_{n}^{k_{j}}(t)=u_{n}^{k_{j}}(0)+\int_{0}^{t}\left(-\nu \lambda^{2 \alpha n} u_{n}^{k_{j}}+\lambda^{n}\left(u_{n-1}^{k_{j}}\right)^{2}-\lambda^{n+1} u_{n}^{k_{j}} u_{n+1}^{k_{j}}+g_{n}\right) d \tau,
$$

for $n \leq k_{j}-1$. Taking the limit as $k_{j} \rightarrow \infty$, we obtain

$$
u_{n}(t)=u_{n}(0)+\int_{0}^{t}\left(-\nu \lambda^{2 \alpha n} u_{n}+\lambda^{n} u_{n-1}^{2}-\lambda^{n+1} u_{n} u_{n+1}+g_{n}\right) d \tau .
$$

Since $u_{n}(t)$ is continuous, it follows that $u_{n} \in C^{1}([0, \infty))$ and satisfies (3.1). 
It remains to prove that $u(t)$ satisfies the energy inequality. Note that $u^{k_{j}}(t)$ satisfies the energy equality

$$
\left|u^{k_{j}}(t)\right|^{2}+2 \nu \int_{t_{0}}^{t}\left\|u^{k_{j}}(\tau)\right\|^{2} d \tau=\left|u^{k_{j}}\left(t_{0}\right)\right|^{2}+2 \int_{t_{0}}^{t}\left(g, u^{k_{j}}(\tau)\right) d \tau,
$$

for all $t \geq t_{0} \geq 0$. Hence, the sequence $\left\{u^{k_{j}}\right\}$ is bounded in $L^{2}\left(\left[t_{0}, t\right] ; V\right)$ for all $t \geq t_{0} \geq 0$. This, together with (4.3), implies that

$$
\int_{t_{0}}^{t}\left|u^{k_{j}}(\tau)-u(\tau)\right|^{2} d \tau \rightarrow 0, \quad \text { as } \quad k_{j} \rightarrow \infty,
$$

for all $t \geq t_{0} \geq 0$. In particular, $\left|u^{k_{j}}(t)\right| \rightarrow|u(t)|$ as $k_{j} \rightarrow \infty$ a.e. in $[0, \infty)$. Take any $t_{0} \geq 0$ for which $\left|u^{k_{j}}\left(t_{0}\right)\right| \rightarrow\left|u\left(t_{0}\right)\right|$ as $k_{j} \rightarrow \infty$. For every $N \geq 0$, we have

$$
\left|u^{k_{j}}(t)\right|^{2}+2 \nu \int_{t_{0}}^{t} \sum_{n \leq N} \lambda^{2 \alpha n} u_{n}^{k_{j}}(\tau)^{2} d \tau \leq\left|u^{k_{j}}\left(t_{0}\right)\right|^{2}+2 \int_{t_{0}}^{t}\left(g, u^{k_{j}}(\tau)\right) d \tau .
$$

Since $u^{k_{j}}(t) \rightarrow u(t)$ weakly in $H$ as $k_{j} \rightarrow \infty$ for all time $t \geq 0$, we have that

$$
|u(t)|^{2}+2 \nu \int_{t_{0}}^{t} \sum_{n \leq N} \lambda^{2 \alpha n} u_{n}(\tau)^{2} d \tau \leq\left|u\left(t_{0}\right)\right|^{2}+2 \int_{t_{0}}^{t}(g, u(\tau)) d \tau .
$$

Finally, taking the limit as $N \rightarrow \infty$ and using Levi's theorem, we obtain

$$
|u(t)|^{2}+2 \nu \int_{t_{0}}^{t}\|u(\tau)\|^{2} d \tau \leq\left|u\left(t_{0}\right)\right|^{2}+2 \int_{t_{0}}^{t}(g, u(\tau)) d \tau,
$$

for all $0 \leq t_{0} \leq t, t_{0}$ a.e. in $[0, \infty)$.

Note that this was a classical proof from the theory of NSE. Using the fact that there is no backward energy transfer, we can actually show that every solution with $u_{n}(0) \geq 0$ is a Leray-Hopf solution and, moreover, is continuous from the right in $H$ for all time.

Theorem 4.2. Let $u(t)$ be a solution of (3.1) with $u_{n}(0) \geq 0$. Then $u_{n}(t) \geq 0$ for all $t>0$, and $u(t)$ satisfies the energy inequality

$$
|u(t)|^{2}+2 \nu \int_{t_{0}}^{t}\|u(\tau)\|^{2} d \tau \leq\left|u\left(t_{0}\right)\right|^{2}+2 \int_{t_{0}}^{t}(g, u(\tau)) d \tau,
$$

for all $0 \leq t_{0} \leq t$.

Proof. A general solution for $u_{n}(t)$ can be written as

$$
\begin{aligned}
u_{n}(t)= & u_{n}(0) \exp \left(-\int_{0}^{t} \nu \lambda^{2 \alpha n}+\lambda^{n+1} u_{n+1}(\tau) d \tau\right) \\
& +\int_{0}^{t} \exp \left(-\int_{s}^{t} \nu \lambda^{2 \alpha n}+\lambda^{n+1} u_{n+1}(\tau) d \tau\right)\left(g_{n}+\lambda^{n} u_{n-1}^{2}(s)\right) d s .
\end{aligned}
$$

Recall that $g_{n} \geq 0$ for all $n$. Since $u_{n}(0) \geq 0$ for all $n$, then $u_{n}(t) \geq 0$ for all $n$, $t>0$. Hence, multiplying (3.1) by $u_{n}$, taking a sum from 1 to $N$, and integrating 
between $t_{0}$ and $t$, we obtain

$$
\begin{aligned}
\sum_{n=1}^{N} u_{n}(t)^{2}- & \sum_{n=1}^{N} u_{n}\left(t_{0}\right)^{2}+2 \nu \int_{t_{0}}^{t} \sum_{n=1}^{N} \lambda^{2 \alpha n} u_{n}(\tau)^{2} d \tau \\
& =-2 \int_{t_{0}}^{t} \lambda^{N+1} u_{N}^{2} u_{N+1} d \tau+2 \int_{t_{0}}^{t} \sum_{n=1}^{N} g_{n} u_{n} d \tau \\
& \leq 2 \int_{t_{0}}^{t} \sum_{n=1}^{N} g_{n} u_{n} d \tau
\end{aligned}
$$

Taking the limit as $N \rightarrow \infty$, we obtain (4.4).

Enstrophy estimates. We obtain the following estimate for the nonlinear term:

$$
\begin{aligned}
|(B(u, u), A u)| & =\left|\sum_{n=1}^{\infty}\left[\lambda^{2 \alpha(n+1)}-\lambda^{2 \alpha n}\right] \lambda^{n} u_{n}^{2} u_{n+1}\right| \\
& =\left(\lambda^{\alpha}-\lambda^{-\alpha}\right)\left|\sum_{n=1}^{\infty} \lambda^{(\alpha+1) n} u_{n}^{2} \lambda^{\alpha(n+1)} u_{n+1}\right| \\
& \leq c_{\mathrm{b}}\left(\max _{n}\left|\lambda^{\alpha n} u_{n}\right|\right) \sum_{n=1}^{\infty} \lambda^{(\alpha+1) n} u_{n}^{2} \\
& \leq c_{\mathrm{b}}\|u\| \sum_{n=1}^{\infty} \lambda^{(\alpha+1) n} u_{n}^{2},
\end{aligned}
$$

where $c_{\mathrm{b}}=\lambda^{\alpha}-\lambda^{-\alpha}>0$. When $\alpha \in[1 / 3,1]$, Hölder's inequality implies

$$
\begin{aligned}
|(B(u, u), A u)| & \leq c_{\mathrm{b}}\|u\||A u|^{1 / \alpha-1}\left|A^{1 / 2} u\right|^{3-1 / \alpha} \\
& =c_{\mathrm{b}}|A u|^{1 / \alpha-1}\|u\|^{4-1 / \alpha} .
\end{aligned}
$$

Choosing $u$ to have only two consecutive nonzero terms, it is easy to check that these estimates are sharp. Moreover, when $\alpha=2 / 5$, we have

$$
|(B(u, u), A u)| \leq c_{\mathrm{b}}|A u|^{3 / 2}\|u\|^{3 / 2},
$$

which is the same as the Sobolev estimate for the inertial term of the 3D NSE (see, e.g., [6, 20]). Therefore, taking a scalar product of the equation (3.1) with $A u$ and using Young's inequality, we obtain

$$
\begin{aligned}
\frac{1}{2} \frac{d}{d t}\|u\|^{2} & \leq-\nu|A u|^{2}+c_{\mathrm{b}}|A u|^{3 / 2}\|u\|^{3 / 2}+(g, A u) \\
& \leq-\nu|A u|^{2}+\frac{\nu}{3}|A u|^{2}+\frac{3^{6} c_{\mathrm{b}}^{4}}{2^{8} \nu^{3}}\|u\|^{6}+\frac{3}{4 \nu}|g|^{2}+\frac{\nu}{3}|A u|^{2} \\
& =-\frac{\nu}{3}|A u|^{2}+\frac{3^{6} c_{\mathrm{b}}^{4}}{2^{8} \nu^{3}}\|u\|^{6}+\frac{3}{4 \nu}|g|^{2}
\end{aligned}
$$

a Riccati-type inequality for $\|u\|^{2}$. Hence, the model has the same enstrophy estimate as the 3D NSE, similar properties, and the same open question concerning the regularity of the solutions in the case $\alpha=2 / 5$.

Another interesting case is $\alpha=1 / 3$. Then we have

$$
|(B(u, u), A u)| \leq c_{\mathrm{b}}|A u|^{2}\|u\|,
$$

which corresponds to the 4D Navier-Stokes equations. 
Now consider the case where $\alpha>1 / 3$. Formally, we have

$$
\begin{aligned}
\frac{1}{2} \frac{d}{d t}\|u\|^{2} & \leq-\nu|A u|^{2}+c_{\mathrm{b}}|A u|^{1 / \alpha-1}\|u\|^{4-1 / \alpha}+(g, A u) \\
& \leq-\nu|A u|^{2}+\frac{\nu}{3}|A u|^{2}+c\|u\|^{\frac{8 \alpha-2}{3 \alpha-1}}+\frac{3}{4 \nu}|g|^{2}+\frac{\nu}{3}|A u|^{2} \\
& =-\frac{\nu}{3}|A u|^{2}+c\|u\|^{\frac{8 \alpha-2}{3 \alpha-1}}+\frac{3}{4 \nu}|g|^{2}
\end{aligned}
$$

for some constant $c>0$. This means that if the initial data is in $V$, then $u(t)$ remains bounded in $V$ for some time $T$. Applying the above estimate to the Galerkin approximation and taking a limit, we immediately obtain the following local regularity result.

Theorem 4.3. If $\alpha>1 / 3$, then for any $u^{0} \in V$ there exists a strong solution $u(t)$ to (3.1) on some time interval $[0, T], T>0$ with $u(0)=u^{0}$.

Finally, consider the case $\alpha \geq 1 / 2$. In this case the enstrophy estimate implies

$$
(B(u, u), A u) \leq c_{\mathrm{b}}|A u|\|u\|^{2} .
$$

Therefore, formally, we have

$$
\begin{aligned}
\frac{1}{2} \frac{d}{d t}\|u\|^{2} & \leq-\nu|A u|^{2}+c_{\mathrm{b}}|A u|\|u\|^{2}+(g, A u) \\
& \leq-\nu|A u|^{2}+\frac{\nu}{3}|A u|^{2}+\frac{3 c_{\mathrm{b}}^{2}}{4 \nu}\|u\|^{4}+\frac{3}{4 \nu}|g|^{2}+\frac{\nu}{3}|A u|^{2} \\
& =-\frac{\nu}{3}|A u|^{2}+\frac{3 c_{\mathrm{b}}^{2}}{4 \nu}\|u\|^{4}+\frac{3}{4 \nu}|g|^{2} .
\end{aligned}
$$

This is again a Riccati-type inequality. Assume that $u(t)$ is a strong solution on some interval $\left(0, t^{*}\right)$, and $\|u(t)\| \rightarrow+\infty$ as $t \rightarrow t^{*}-$. Then

$$
\|u(t)\|^{2} \geq \frac{c}{t^{*}-t}, \quad 0<t<t^{*}
$$

for some positive constant $c$. However, this means that $\|u(t)\|^{2}$ is not locally integrable, which is in contradiction with the energy inequality. Hence, if the initial data $u^{0} \in V$, then $\|u(t)\|$ is bounded on every interval $[0, T], T>0$, and we have the following.

Theorem 4.4. If $\alpha \geq 1 / 2$, then for any $u^{0} \in V$ there exists a strong solution $u(t)$ to (3.1) on $[0, \infty)$ with $u(0)=u^{0}$.

\section{BlOW-UP IN FINITE TIME}

Let $\alpha<1 / 3$ and $\gamma>0$. In this section we will prove that every solution $u(t)$ with large enough $\|u(0)\|_{\gamma}$ blows up in finite time in the $H^{1 / 3+\gamma}$ norm. The idea is the following. Taking a scalar product of the equation with $A^{\gamma / \alpha} u$, we obtain

$$
\frac{1}{2} \frac{d}{d t}\|u\|_{\gamma}^{2}=-\nu\|u\|_{\alpha+\gamma}^{2}+\left(B(u, u), A^{\gamma / \alpha} u\right)+\left(g, A^{\gamma / \alpha} u\right) .
$$


In order to show a blow-up, we, in some sense, will invert the Sobolev estimates for the nonlinear term. Note that

$$
\left(B(u, u), A^{\gamma / \alpha} u\right) \sim \sum_{n} \lambda^{(1+2 \gamma) n} u_{n}^{2} u_{n+1} .
$$

If $u_{n} \geq 0$ is monotonically decreasing in $n$, then

$$
\left(B(u, u), A^{\gamma / \alpha} u\right) \gtrsim \sum_{n} \lambda^{(1+2 \gamma) n} u_{n}^{3} .
$$

Obviously, this is not true in general. For example, if $u_{n}=0$ for even $n$, then $\left(B(u, u), A^{\gamma / \alpha} u\right)=0$. However, we will prove that a similar estimate holds if we use the following function instead of the $H^{\gamma}$-norm:

$$
H(t):=\|u(t)\|_{\gamma}^{2}+c \sum_{n} \lambda^{2 \gamma n}\left(u_{n} u_{n+1}\right)(t)
$$

for some constant $c>0$. More precisely, we will show that if $\|u\|_{\gamma}$ is large enough, then

$$
\begin{aligned}
\frac{1}{2} \frac{d}{d t} H & \gtrsim-\nu\|u\|_{\alpha+\gamma}^{2}+\sum_{n} \lambda^{(1+2 \gamma) n} u_{n}^{3} \\
& \gtrsim-\nu\|u\|_{\alpha+\gamma}^{2}+\|u\|_{\alpha+\gamma}^{3} \\
& \gtrsim H^{3 / 2},
\end{aligned}
$$

provided that $\alpha<1 / 3$. We will start with the following estimate.

Lemma 5.1. If $\alpha<1 / 3$, then for any $\gamma \in(0,1-3 \alpha)$ there exists a positive constant A such that

$$
\sum_{n=1}^{\infty} \lambda^{(1+2 \gamma) n}\left|u_{n}\right|^{3} \geq A\|u\|_{\alpha+\gamma}^{3} .
$$

Proof. Let $\epsilon:=2-6 \alpha-2 \gamma>0$. Note that $\lambda^{-\epsilon}<1$. Let

$$
A(\gamma):=\left(\sum_{n=1}^{\infty} \lambda^{-\epsilon n}\right)^{-1 / 2}=\sqrt{\lambda^{\epsilon}-1}
$$

Hölder's inequality with $p=3$ and $q=3 / 2$ implies

$$
\begin{aligned}
\|u\|_{\alpha+\gamma}^{2} & =\sum_{n=1}^{\infty} \lambda^{2(\alpha+\gamma) n} u_{n}^{2} \\
& \leq\left(\sum_{n=1}^{\infty} \lambda^{-\epsilon n}\right)^{1 / 3}\left(\sum_{n=1}^{\infty} \lambda^{(1+2 \gamma) n}\left|u_{n}\right|^{3}\right)^{2 / 3} \\
& =A^{-2 / 3}\left(\sum_{n=1}^{\infty} \lambda^{(1+2 \gamma) n}\left|u_{n}\right|^{3}\right)^{2 / 3}
\end{aligned}
$$

Hence,

$$
\sum_{n=1}^{\infty} \lambda^{(1+2 \gamma) n}\left|u_{n}\right|^{3} \geq A\|u\|_{\alpha+\gamma}^{3}
$$

which concludes the proof. 
Lemma 5.2. Let $u(t)$ be a solution to (3.1) and $\gamma>0$. Let $\|u(t)\|_{\gamma}^{2}$ be continuous on $[0, \infty)$. Then the function

$$
\sum_{n=1}^{\infty} \lambda^{2 \gamma n}\left(u_{n} u_{n+1}\right)(t)
$$

is continuous on $[0, \infty)$.

Proof. Let $v_{n}(t)=\lambda^{2 \gamma n}\left(u_{n} u_{n+1}\right)(t)$. First, note that due to the Cauchy-Schwarz inequality, the function (5.1) is less than or equal to $\|u(t)\|_{\gamma}^{2}$ and, consequently, is bounded on every interval $[a, b], 0 \leq a<b$. Let $t_{0}>0$. Since $\|u(t)\|_{\gamma}^{2}$ is continuous at $t=t_{0}$, it follows that

$$
\lim _{N \rightarrow \infty} \limsup _{t \rightarrow t_{0}} \sum_{n=N}^{\infty} \lambda^{2 \gamma n} u_{n}^{2}(t)=0 .
$$

Therefore,

$$
\lim _{N \rightarrow \infty} \limsup _{t \rightarrow t_{0}} \sum_{n=N}^{\infty} v_{n}(t)=0
$$

which means that (5.1) is continuous at $t=t_{0}$. Indeed, since $v_{n}(t)$ is continuous for every $n$, we have

$$
\begin{aligned}
\limsup _{t \rightarrow t_{0}} \mid & \left|\sum_{n=1}^{\infty} v_{n}(t)-\sum_{n=1}^{\infty} v_{n}\left(t_{0}\right)\right| \\
& =\lim _{N \rightarrow \infty} \limsup _{t \rightarrow t_{0}}\left|\sum_{n=1}^{N-1} v_{n}(t)-\sum_{n=1}^{N-1} v_{n}\left(t_{0}\right)+\sum_{n=N}^{\infty} v_{n}(t)-\sum_{n=N}^{\infty} v_{n}\left(t_{0}\right)\right|=0 .
\end{aligned}
$$

Similarly, the continuity of (5.1) from the right holds at $t=0$.

Now we proceed to our main result.

Theorem 5.3. Let $u(t)$ be a solution to (3.1) with $u_{n}(0) \geq 0$ and $\alpha<1 / 3$. Then for every $\gamma>0$, there exists a constant $M(\gamma)$, such that $\|u(t)\|_{1 / 3+\gamma}^{3}$ is not locally integrable on $[0, \infty)$, provided $\|u(0)\|_{\gamma}>M(\gamma)$.

Proof. Since $\|u\|_{\gamma_{1}} \leq\|u\|_{\gamma_{2}}$ for $\gamma_{1} \leq \gamma_{2}$, it is enough to prove the theorem in the case $0<\gamma<\min \{1 / 3,1-3 \alpha\}$. Given such a $\gamma$, let $u(t)$ be a solution to (3.1) such that $\|u(t)\|_{1 / 3+\gamma}^{3}$ is integrable on $[0, T]$ for every $T>0$. We will show that $\|u(0)\|_{\gamma}$ is bounded from above by a constant dependent on $\gamma$.

Note that $u_{n}(t) \geq 0$ for all $n, t>0$ due to Theorem 4.2. First, we obtain

$$
\begin{aligned}
\int_{0}^{T} \sum_{n=1}^{\infty} \lambda^{(1+2 \gamma) n} u_{n}^{2} u_{n+1} d \tau & \leq \int_{0}^{T} \sum_{n=1}^{\infty} \lambda^{(1+2 \gamma) n}\left(u_{n}^{3}+u_{n+1}^{3}\right) d \tau \\
& \leq 2 \int_{0}^{T}\left(\sum_{n=1}^{\infty} \lambda^{\frac{2}{3}(1+2 \gamma) n} u_{n}^{2}\right)^{3 / 2} d \tau \\
& \leq 2 \int_{0}^{T}\|u(t)\|_{1 / 3+\gamma}^{3} d \tau \\
& <\infty
\end{aligned}
$$


for all $T>0$. Thus,

$$
\sum_{n=1}^{\infty} \lambda^{(1+2 \gamma) n}\left(u_{n}^{2} u_{n+1}\right)(t) \quad \text { and } \quad \sum_{n=1}^{\infty} \lambda^{(1+2 \gamma) n} u_{n}(t)^{3}
$$

are locally integrable on $[0, \infty)$. In addition, since $\alpha<1 / 3$, we have

$$
\|u(t)\|_{\alpha+\gamma}^{2} \leq\|u(t)\|_{1 / 3+\gamma}^{2},
$$

which implies that $\|u(t)\|_{\alpha+\gamma}^{2}$ is locally integrable on $[0, \infty)$.

Now note that if $u_{n} \leq \frac{1}{2} u_{n+1}$, then $u_{n} u_{n+1}^{2} \leq \frac{1}{2} u_{n+1}^{3}$. Otherwise, $u_{n} u_{n+1}^{2} \leq$ $2 u_{n}^{2} u_{n+1}$. Hence,

$$
u_{n} u_{n+1}^{2} \leq \frac{1}{2} u_{n+1}^{3}+2 u_{n}^{2} u_{n+1}, \quad n \in \mathbb{N} .
$$

This also implies that

$$
\begin{aligned}
u_{n} u_{n+1} u_{n+2} & \leq \frac{1}{2} u_{n}^{2} u_{n+1}+\frac{1}{2} u_{n+1} u_{n+2}^{2} \\
& \leq \frac{1}{2} u_{n}^{2} u_{n+1}+\frac{1}{4} u_{n+2}^{3}+u_{n+1}^{2} u_{n+2},
\end{aligned}
$$

for all $n \in \mathbb{N}$.

From (3.1) we obtain

$$
\begin{aligned}
\frac{d}{d t}\left(u_{n} u_{n+1}\right)= & -\nu\left(\lambda^{2 \alpha n}+\lambda^{2 \alpha(n+1)}\right) u_{n} u_{n+1} \\
& +\lambda^{n} u_{n-1}^{2} u_{n+1}-\lambda^{n+1} u_{n} u_{n+1}^{2} \\
& +\lambda^{n+1} u_{n}^{3}-\lambda^{n+2} u_{n} u_{n+1} u_{n+2} \\
& +g_{n} u_{n+1}+g_{n+1} u_{n} .
\end{aligned}
$$

This, together with inequalities (5.3) and (5.4), implies that

$$
\begin{aligned}
\frac{d}{d t}\left(u_{n} u_{n+1}\right)+ & \nu\left(1+\lambda^{2 \alpha}\right) \lambda^{2 \alpha n} u_{n} u_{n+1} \\
& +2 \lambda^{n+1} u_{n}^{2} u_{n+1}+\frac{1}{2} \lambda^{n+2} u_{n}^{2} u_{n+1}+\lambda^{n+2} u_{n+1}^{2} u_{n+2} \\
& \geq \lambda^{n+1} u_{n}^{3}-\frac{1}{2} \lambda^{n+1} u_{n+1}^{3}-\frac{1}{4} \lambda^{n+2} u_{n+2}^{3} .
\end{aligned}
$$

Multiplying this by $\lambda^{2 \gamma n}$, taking a sum from 1 to $\infty$, and integrating between 0 and $t$, we get

$$
\begin{aligned}
\sum_{n=1}^{\infty} \lambda^{2 \gamma n}\left(u_{n} u_{n+1}\right)(t)-\sum_{n=1}^{\infty} \lambda^{2 \gamma n}\left(u_{n} u_{n+1}\right)(0) \\
+\nu\left(1+\lambda^{2 \alpha}\right) \int_{0}^{t} \sum_{n=1}^{\infty} \lambda^{2(\alpha+\gamma) n} u_{n} u_{n+1} d \tau \\
+\left(2 \lambda+\frac{1}{2} \lambda^{2}+\lambda^{1-2 \gamma}\right) \int_{0}^{t} \sum_{n=1}^{\infty} \lambda^{(1+2 \gamma) n} u_{n}^{2} u_{n+1} d \tau \\
\geq\left(\lambda-\frac{1}{2} \lambda^{-2 \gamma}-\frac{1}{4} \lambda^{-4 \gamma}\right) \int_{0}^{t} \sum_{n=1}^{\infty} \lambda^{(1+2 \gamma) n} u_{n}^{3} d \tau \\
\geq \frac{\lambda}{4} \int_{0}^{t} \sum_{n=1}^{\infty} \lambda^{(1+2 \gamma) n} u_{n}^{3} d \tau
\end{aligned}
$$


for all $t \geq 0$. On the other hand, we have the following equality for the nonlinear term:

$$
\begin{aligned}
-\left(B(u, u), A^{\gamma / \alpha} u\right) & =\sum_{n=1}^{\infty} \lambda^{2 \gamma n+n} u_{n-1}^{2} u_{n}-\sum_{n=1}^{\infty} \lambda^{2 \gamma n+n+1} u_{n}^{2} u_{n+1} \\
& =c_{1} \sum_{n=1}^{\infty} \lambda^{(1+2 \gamma) n} u_{n}^{2} u_{n+1},
\end{aligned}
$$

where $c_{1}=\lambda^{2 \gamma+1}-\lambda>0$. Now, multiplying (3.1) by $\lambda^{2 \gamma n} u_{n}$, taking a sum from 1 to $\infty$, and integrating between 0 and $t$, we obtain

$$
\begin{aligned}
\|u(t)\|_{\gamma}^{2}-\|u(0)\|_{\gamma}^{2} & +2 \nu \int_{0}^{t}\|u(\tau)\|_{\alpha+\gamma}^{2} d \tau \\
& =2 c_{1} \int_{0}^{t} \sum_{n=1}^{\infty} \lambda^{(1+2 \gamma) n} u_{n}^{2} u_{n+1} d \tau+2 \int_{0}^{t} \sum_{n=1}^{\infty} \lambda^{2 \gamma n} g_{n} u_{n} d \tau
\end{aligned}
$$

for $t \geq 0$. Note that the term with the force is integrable because $\gamma \leq 1 / 3$. In particular, (5.6) yields that $\|u(t)\|_{\gamma}^{2}$ is continuous on $[0, \infty)$. Denote

$$
H(t):=\|u(t)\|_{\gamma}^{2}+c_{2} \sum_{n=1}^{\infty} \lambda^{2 \gamma n}\left(u_{n} u_{n+1}\right)(t),
$$

where $c_{2}=2 c_{1} /\left(2 \lambda+\lambda^{2} / 2+\lambda^{1-2 \gamma}\right)$. Thanks to Lemma 5.2. $H(t)$ is continuous on $[0, \infty)$. We will show that $H(t)$ is a Lyapunov function, i.e., $H(t)$ is always increasing. Moreover, we will see that $H(t)$ blows up in finite time. Indeed, multiplying (5.5) by $c_{2}$ and adding (5.6), we get

$$
\begin{aligned}
H(t)-H(0) \geq-2 \nu \int_{0}^{t}\|u(\tau)\|_{\alpha+\gamma}^{2} d \tau-\nu c_{3} \int_{0}^{t} \sum_{n=1}^{\infty} \lambda^{2(\alpha+\gamma) n} u_{n} u_{n+1} d \tau \\
+\frac{\lambda c_{2}}{4} \int_{0}^{t} \sum_{n=1}^{\infty} \lambda^{(1+2 \gamma) n} u_{n}^{3} d \tau
\end{aligned}
$$

where $c_{3}=\left(1+\lambda^{2 \alpha}\right) c_{2}$. Due to Lemma 5.1, there exists a constant $A>0$ such that

$$
\sum_{n=1}^{\infty} \lambda^{(1+2 \gamma) n} u_{n}^{3} \geq A\|u\|_{\alpha+\gamma}^{3} .
$$

In addition, the Cauchy-Schwarz inequality implies

$$
\sum_{n=1}^{\infty} \lambda^{2(\alpha+\gamma) n} u_{n} u_{n+1} \leq\|u\|_{\alpha+\gamma}^{2} .
$$

Therefore, we obtain

$$
H(t)-H(0) \geq-\nu\left(2+c_{3}\right) \int_{0}^{t}\|u(\tau)\|_{\alpha+\gamma}^{2} d \tau+\frac{A \lambda c_{2}}{4} \int_{0}^{t}\|u(\tau)\|_{\alpha+\gamma}^{3} d \tau,
$$

for $t \geq 0$. Note that

$$
\|u(t)\|_{\gamma}^{2} \leq H(t) \leq\left(1+c_{2}\right)\|u(t)\|_{\gamma}^{2}
$$


In particular,

Let

$$
\|u(t)\|_{\alpha+\gamma} \geq \sqrt{\frac{H(t)}{1+c_{2}}} .
$$

$$
M(\gamma):=\frac{8 \nu\left(2+c_{3}\right) \sqrt{1+c_{2}}}{A \lambda c_{2}} .
$$

Assume that $H(\tau) \geq M^{2}$ on $[0, t]$ for some $t>0$. Then we have that $\|u(\tau)\|_{\alpha+\gamma} \geq$ $8 \nu\left(2+c_{3}\right) /\left(A \lambda c_{2}\right)$ on $[0, t]$ and, consequently, (5.7) yields

$$
H(t)-H(0) \geq c \int_{0}^{t} H(\tau)^{3 / 2} d \tau,
$$

where $c=A \lambda c_{2} / 8$. Now assume that $\|u(0)\|_{\gamma}>M$. Then $H(0)>M^{2}$ and (5.8) holds for some small time $t>0$. Then (5.8) automatically holds for every $t>0$.

Note that (5.8) is a Riccati-type inequality. It is easy to see that $H(t)$ blows up in finite time. Indeed, let $y(t)$ be the solution to the Riccati equation

$$
y^{\prime}(t)=c y(t)^{3 / 2}, \quad y(0)=\frac{1}{2} H(0) .
$$

Then for some $t^{*}>0$, we have that $y(t) \rightarrow \infty$ as $t \rightarrow t^{*}-$. Consider

$$
w(t)=H(t)-y(t) .
$$

It is easy to check that the function $w(t)$ satisfies the following integral inequality:

$$
w(t)-w(0) \geq c \int_{0}^{t} w(\tau)^{3 / 2} d \tau,
$$

for all $t>0$, such that $w(\tau) \geq 0$ on $[0, t]$. Note that $w(0)>0$ and $w(t)$ is continuous. Thus, $w(t) \geq 0$ for all $t \in\left[0, t^{*}\right)$.

Now, since $y(t)$ blows up in finite time, $H(t)$ also blows up in finite time, which contradicts the fact that $H(t)$ is continuous on $[0, \infty)$. Hence, $\|u(0)\|_{\gamma} \leq M$.

\section{Global attractor}

In Section 4 we showed that the dyadic model possesses an absorbing ball with a radius $R$ larger than $|g| / \nu$. Let $X$ be a closed absorbing ball,

$$
X:=\{u \in H:|u| \leq R\},
$$

which is compact in the $\mathrm{d}_{\mathrm{w}}$-metric. Then for any bounded set $K \subset H$, there exists a time $t_{0}$ such that

$$
u(t) \in X, \quad \forall t \geq t_{0},
$$

for every Leray-Hopf solution $u(t)$ to (3.1) with the initial data $u(0) \in K$.

We recall the definition of an evolutionary system $\mathcal{E}$ from [4] (see also [3]). Let

$$
\mathcal{T}:=\{I: I=[T, \infty) \subset \mathbb{R} \text {, or } I=(-\infty, \infty)\},
$$

and for each $I \subset \mathcal{T}$ let $\mathcal{F}(I)$ denote the set of all $X$-valued functions on $I$. A map $\mathcal{E}$ that associates to each $I \in \mathcal{T}$ a subset $\mathcal{E}(I) \subset \mathcal{F}$ will be called an evolutionary system if the following conditions are satisfied:

(1) $\mathcal{E}([0, \infty)) \neq \emptyset$.

(2) $\mathcal{E}(I+s)=\{u(\cdot): u(\cdot-s) \in \mathcal{E}(I)\}$ for all $s \in \mathbb{R}$.

(3) $\left\{\left.u(\cdot)\right|_{I_{2}}: u(\cdot) \in \mathcal{E}\left(I_{1}\right)\right\} \subset \mathcal{E}\left(I_{2}\right)$ for all pairs of $I_{1}, I_{2} \in \Omega$ such that $I_{2} \subset I_{1}$.

(4) $\mathcal{E}((-\infty, \infty))=\left\{u(\cdot):\left.u(\cdot)\right|_{[T, \infty)} \in \mathcal{E}([T, \infty)) \forall T \in \mathbb{R}\right\}$. 
Let

$$
\begin{aligned}
R(t) A & :=\{u(t): u(0) \in A, u \in \mathcal{E}([0, \infty))\}, \\
\widetilde{R}(t) A & :=\{u(t): u(0) \in A, u \in \mathcal{E}((-\infty, \infty))\}, \quad A \subset X, t \geq 0 .
\end{aligned}
$$

For $A \subset X$ and $r>0$, denote $B \bullet(A, r)=\left\{u: d_{\bullet}(A, u)<r\right\}$, where $\bullet=\mathrm{s}$, w. Now we define an attracting set and a global attractor as follows.

Definition 6.1. A set $A \subset X$ is a d.-attracting set $(\bullet=\mathrm{s}, \mathrm{w})$ if it uniformly attracts $X$ in the $\mathrm{d}_{\bullet}$-metric; i.e., for any $\epsilon>0$ there exists $t_{0}$ such that

$$
R(t) X \subset B_{\bullet}(A, \epsilon), \quad \forall t \geq t_{0} .
$$

A set $A \subset X$ is invariant if $\widetilde{R}(t) A=A$ for all $t \geq 0$. A set $\mathcal{A}_{\bullet} \subset X$ is a d.-global attractor if $\mathcal{A}_{\bullet}$ is a minimal $\mathrm{d}_{\bullet}$-closed $\mathrm{d}_{\bullet}$-attracting set.

The following result was proved in [4]:

Theorem 6.2. The evolutionary system $\mathcal{E}$ always possesses a weak global attractor $\mathcal{A}_{\mathrm{w}}$. In addition, if $\mathcal{E}([0, \infty))$ is compact in $C\left([0, \infty) ; H_{\mathrm{w}}\right)$, then

(a) $\mathcal{A}_{\mathrm{w}}=\left\{u^{0}: u^{0}=u(0)\right.$ for some $\left.u \in \mathcal{E}((-\infty, \infty))\right\}$,

(b) $\mathcal{A}_{\mathrm{w}}$ is the maximal invariant set.

For the dyadic model, we define $\mathcal{E}$ in the following way:

$$
\begin{aligned}
\mathcal{E}([T, \infty)):=\{u: u(\cdot) \text { is a Leray-Hopf solution on }[T, \infty) & \text { and } u(t) \in X \forall t \in[T, \infty)\}, \quad T \in \mathbb{R}, \\
\mathcal{E}((\infty, \infty)):=\{u: u(\cdot) \text { is a Leray-Hopf solution on }(-\infty, \infty) & \text { and } u(t) \in X \forall t \in(-\infty, \infty)\},
\end{aligned}
$$

where $X$ is the phase space defined in the beginning of the section. Clearly, $\mathcal{E}$ satisfies properties (1)-(4). Then Theorem 6.2 immediately yields that the weak global attractor $\mathcal{A}_{\mathrm{w}}$ exists. In order to infer that $\mathcal{A}_{\mathrm{w}}$ is the maximal invariant set, we need the following result.

Lemma 6.3. $\mathcal{E}([0, \infty))$ is compact in $C\left([0, \infty) ; H_{\mathrm{w}}\right)$.

Proof. Take any sequence $u^{k} \in \mathcal{E}([0, \infty))$. First, note that

$$
u_{n}^{k}(t) \leq R, \quad \forall n, k, t \geq 0 .
$$

Therefore,

$$
\left|u_{n}^{k}(t)-u_{n}^{k}(s)\right| \leq\left(\nu \lambda^{2 \alpha n} R+\lambda^{n} R^{2}+\lambda^{n+1} R^{2}+g_{n}\right)|t-s|,
$$

for all $n, k, t \geq 0, s \geq 0$. Thus,

$$
\mathrm{d}_{\mathrm{w}}\left(u^{k}(t), u^{k}(s)\right)=\sum_{n=1}^{\infty} \frac{1}{2^{\left(n^{2}\right)}} \frac{\left|u_{n}^{k}(t)-u_{n}^{k}(s)\right|}{1+\left|u_{n}^{k}(t)-u_{n}^{k}(s)\right|} \leq c|t-s|,
$$

for some constant $c$ independent of $k$. Hence, $\left\{u^{k}\right\}$ is an equicontinuous sequence of functions in $C\left([0, \infty) ; H_{\mathrm{w}}\right)$ with bounded initial data. Therefore, the AscoliArzela theorem implies that $\left\{u^{k}\right\}$ is relatively compact in $C\left([0, T] ; H_{\mathrm{w}}\right)$ for all $T>$ 0 . Using a diagonalization process, we obtain that $\left\{u^{k}\right\}$ is relatively compact in $C\left([0, \infty) ; H_{\mathrm{w}}\right)$. Hence, there exists a weakly continuous $H$-valued function $u(t)$ on $[0, \infty)$ such that

$$
u^{k_{j}} \rightarrow u \quad \text { as } \quad k_{j} \rightarrow \infty \quad \text { in } \quad C\left([0, \infty) ; H_{\mathrm{w}}\right),
$$


for some subsequence $k_{j}$. In particular,

$$
|u(t)| \leq \liminf _{k_{j} \rightarrow \infty}\left|u^{k_{j}}(t)\right| \leq R, \quad t \geq 0,
$$

i.e., $u(t) \in X$ for all $t \geq 0$.

In addition, since $u^{\bar{k}_{j}}(t)$ is a solution to (3.1), we have

$$
u_{n}^{k_{j}}(t)=u_{n}^{k_{j}}(0)+\int_{0}^{t}\left(-\nu \lambda^{2 \alpha n} u_{n}^{k_{j}}+\lambda^{n}\left(u_{n-1}^{k_{j}}\right)^{2}-\lambda^{n+1} u_{n}^{k_{j}} u_{n+1}^{k_{j}}+g_{n}\right) d \tau,
$$

for all $n$. Taking the limit as $k_{j} \rightarrow \infty$, we obtain

$$
u_{n}(t)=u_{n}(0)+\int_{0}^{t}\left(-\nu \lambda^{2 \alpha n} u_{n}+\lambda^{n} u_{n-1}^{2}-\lambda^{n+1} u_{n} u_{n+1}+g_{n}\right) d \tau,
$$

for all $n$. Since $u_{n}(t)$ is continuous, $u_{n} \in C^{1}([0, \infty))$ and satisfies (3.1).

In order to infer that $u \in \mathcal{E}([0, \infty))$, it remains to prove that $u(t)$ satisfies the energy inequality. Note that $\left|u^{k_{j}}(t)\right| \rightarrow|u(t)|$ as $k_{j} \rightarrow \infty$ a.e. in $[0, \infty)$. Since $u^{k_{j}} \in \mathcal{E}([0, \infty))$, it satisfies the energy inequality starting from any $t_{0}$ that is not in the exceptional set of measure zero. Let $E x$ be the union of the exceptional sets for all $u^{k_{j}}$. Note that $E x$ is of measure zero. Take any $t_{0} \notin E x$ for which $\left|u^{k_{j}}\left(t_{0}\right)\right| \rightarrow\left|u\left(t_{0}\right)\right|$ as $k_{j} \rightarrow \infty$. Then

$$
\left|u^{k_{j}}(t)\right|^{2}+2 \nu \int_{t_{0}}^{t}\left\|u^{k_{j}}(\tau)\right\|^{2} d \tau \leq\left|u^{k_{j}}\left(t_{0}\right)\right|^{2}+2 \int_{t_{0}}^{t}\left(g, u^{k_{j}}(\tau)\right) d \tau,
$$

for all $t \geq t_{0}$. Hence,

$$
\left|u^{k_{j}}(t)\right|^{2}+2 \nu \int_{t_{0}}^{t} \sum_{n \leq N} \lambda^{2 \alpha n} u_{n}^{k_{j}}(\tau)^{2} d \tau \leq\left|u^{k_{j}}\left(t_{0}\right)\right|^{2}+2 \int_{t_{0}}^{t}\left(g, u^{k_{j}}(\tau)\right) d \tau .
$$

Since $u^{k_{j}}(t) \rightarrow u(t)$ weakly in $H$ as $k_{j} \rightarrow \infty$ for all time $t \geq 0$, we have that

$$
|u(t)|^{2}+2 \nu \int_{t_{0}}^{t} \sum_{n \leq N} \lambda^{2 \alpha n} u_{n}(\tau)^{2} d \tau \leq\left|u\left(t_{0}\right)\right|^{2}+2 \int_{t_{0}}^{t}(g, u(\tau)) d \tau .
$$

Finally, taking the limit as $N \rightarrow \infty$ and using Levi's convergence theorem, we obtain

$$
|u(t)|^{2}+2 \nu \int_{t_{0}}^{t}\|u(\tau)\|^{2} d \tau \leq\left|u\left(t_{0}\right)\right|^{2}+2 \int_{t_{0}}^{t}(g, u(\tau)) d \tau,
$$

for all $0 \leq t_{0} \leq t, t_{0}$ a.e. in $[0, \infty)$. Hence, $u \in \mathcal{E}([0, \infty))$, which concludes the proof.

Now Theorem 6.2 implies that the weak global attractor $\mathcal{A}_{\mathrm{w}}$ is the maximal invariant set that consists of the points that belong to complete trajectories, i.e., trajectories in $\mathcal{E}((\infty, \infty))$. Moreover, using (4.5), one can show that $u_{n} \geq 0$ for every $u \in \mathcal{A}_{\mathrm{w}}$. Consider now the case $\alpha<1 / 3$. It is easy to show that for every $\gamma \in(0,1-3 \alpha)$, we can take $g_{1}$ large enough, so that for every solution $u(t)$ and every $t \geq 0$, we have $|u(\tau)|>M(\gamma)$ for some $\tau \in[t, t+1]$. Thanks to Theorem 5.3. this means that $\mathcal{A}_{\mathrm{w}}$ is not bounded in $H^{1 / 3+\gamma}$. It is an open question whether $\mathcal{A}_{\mathrm{w}}$ is bounded in $V$.

We will now proceed to study the question whether $\mathcal{A}_{\mathrm{w}}$ is also a strong global attractor. 
Theorem 6.4. Let $\alpha \geq 1 / 2$. Then every Leray-Hopf solution $u(t)$ of (3.1) satisfies the energy equality

$$
|u(t)|^{2}+2 \nu \int_{t_{0}}^{t}\|u(\tau)\|^{2} d \tau=\left|u\left(t_{0}\right)\right|^{2}+2 \int_{t_{0}}^{t}(g, u(\tau)) d \tau, \quad 0 \leq t_{0} \leq t .
$$

Proof. Let $u(t)$ be a Leray-Hopf solution of (3.1). Thanks to the energy inequality (4.4), $\|u(t)\|^{2}$ is locally integrable. Then we obtain

$$
\begin{aligned}
\int_{t_{0}}^{t} \sum_{n=1}^{\infty} \lambda^{n} u_{n}^{2} u_{n+1} d \tau & \leq \int_{t_{0}}^{t} \sum_{n=1}^{\infty} \lambda^{n}\left(u_{n}^{3}+u_{n+1}^{3}\right) d \tau \\
& \leq 2 \sup _{s \in\left[t_{0}, t\right]}|u(s)| \int_{t_{0}}^{t} \sum_{n=1}^{\infty} \lambda^{n} u_{n}^{2} d \tau \\
& \leq 2 R \int_{t_{0}}^{t}\|u(\tau)\|^{2} d \tau \\
& <\infty
\end{aligned}
$$

for $0 \leq t_{0} \leq t$. Hence,

$$
\int_{t_{0}}^{t} \lambda^{n+1} u_{n}^{2} u_{n+1} d \tau \rightarrow 0, \quad \text { as } \quad n \rightarrow \infty .
$$

Multiplying (3.1) by $u_{n}$, taking a sum from 1 to $N$, and integrating between $t_{0}$ and $t$, we obtain

$$
\begin{aligned}
\sum_{n=1}^{N} u_{n}(t)^{2}-\sum_{n=1}^{N} u_{n}\left(t_{0}\right)^{2}+2 \nu \int_{t_{0}}^{t} \sum_{n=1}^{N} \lambda^{2 \alpha n} u_{n}(\tau)^{2} d \tau \\
=-2 \int_{t_{0}}^{t} \lambda^{N+1} u_{N}^{2} u_{N+1} d \tau+2 \int_{t_{0}}^{t} \sum_{n=1}^{N} g_{n} u_{n}
\end{aligned}
$$

Finally, taking the limit as $N \rightarrow \infty$, we arrive at

$$
|u(t)|^{2}+2 \nu \int_{t_{0}}^{t}\|u(\tau)\|^{2} d \tau=\left|u\left(t_{0}\right)\right|^{2}+2 \int_{t_{0}}^{t}(g, u(\tau)) d \tau, \quad 0 \leq t_{0} \leq t .
$$

In [4] it was proved that the asymptotic compactness of the dynamical system $\mathcal{E}$ implies that the strong global attractor $\mathcal{A}_{\mathrm{s}}$ exists, is strongly compact, and coincides with $\mathcal{A}_{\mathrm{w}}$. In the case where the evolutionary system consists of the Leray-Hopf weak solutions to the 3D NSE, the continuity of the complete trajectories, i.e. trajectories on $\mathcal{A}_{\mathrm{w}}$, implies the asymptotic compactness of $\mathcal{E}$ (see also [1] and [19] for similar results). In 3 this result was proved for an abstract evolutionary system satisfying the energy inequality. It immediately implies the following.

Corollary 6.5. Let $\alpha \geq 1 / 2$. Then $\mathcal{A}_{\mathrm{w}}$ is a strongly compact strong global attractor. 
Note that if $\alpha \geq 1 / 2$, then, thanks to Theorem 4.4 for every initial datum in $H$ there exists a regular solution on $[0, \infty)$. Moreover, it can be shown that such a solution is unique in the class of all Leray-Hopf solutions. Hence, Corollary 6.5 can also be obtained using a classical theory of semiflows. It is an open question whether the continuity of the complete trajectories and, consequently, the existence of the strong compact global attractor holds for $\alpha<1 / 2$.

\section{ACKNOWLEDGMENT}

The author would like to thank Susan Friedlander and the anonymous referee for helpful comments and suggestions.

\section{REFERENCES}

[1] J. M. Ball, Continuity properties and global attractors of generalized semiflows and the Navier-Stokes equations, J. Nonlinear Sci. 7 (1997), 475-502. Erratum: J. Nonlinear Sci. 8 (1998), 233. MR1462276 (98j:58071a), MR1606601 (98j:58071b)

[2] L. Biferale, Shell models of energy cascade in turbulence, Annu. Rev. Fluid Mech. 35 (2003), 441-468. MR.1967019 (2004b:76074)

[3] A. Cheskidov, Global attractors of evolutionary systems, preprint, (2006).

[4] A. Cheskidov and C. Foias, On global attractors of the 3D Navier-Stokes equations, J. Differential Equations 231 (2006), 714-754. MR2287904

[5] A. Cheskidov, S. Friedlander, and N. Pavlović, An inviscid dyadic model of turbulence: the global attractor, preprint, (2006).

[6] P. Constantin and C. Foias, Navier-Stokes Equation, The University of Chicago Press, Chicago, 1989. MR972259 (90b:35190)

[7] P. Constantin, B. Levant, and E. Titi, Analytic study of shell models of turbulence, Phys. D. 219 (2006), 120-141. MR 2251486 (2007h:76055)

[8] E. I. Dinaburg and Ya. G. Sinai, A quasi-linear approximation of three-dimensional NavierStokes system, Moscow Math. J. 1 (2001), 381-388. MR1877599(2002i:76035)

[9] S. Friedlander and N. Pavlović, Blow up in a three-dimensional vector model for the Euler equations, Comm. Pure Appl. Math. 57 (2004), 705-725. MR2038114 (2005c:35241)

[10] S. Friedlander and N. Pavlović, Remarks concerning modified Navier-Stokes equations, Discrete and Continuous Dynamical Systems 10 (2004), 269-288. MR.2026195 (2005a:76043)

[11] U. Frisch, Turbulence. The Legacy of A. N. Kolmogorov, Cambridge University Press, Cambridge, 1995. MR1428905 (98e:76002)

[12] E. B. Gledzer, System of hydrodynamic type admitting two quadratic integrals of motion, Soviet Phys. Dokl. 18 (1973), 216-217.

[13] N. H. Katz and N. Pavlović, A cheap Caffarelli-Kohn-Nirenberg inequality for the NavierStokes equation with hyper-dissipation, Geom. Funct. Anal. 12 (2002), 355-379. MR1911664 (2003e:35243)

[14] N. H. Katz and N. Pavlović, Finite time blow-up for a dyadic model of the Euler equations, Trans. Amer. Math. Soc. 357 (2005), 695-708. MR2095627 (2005h:35284)

[15] A. Kiselev and A. Zlatoš, On discrete models of the Euler equation, IMRN 38 (2005), 23152339. MR2180809(2007e:35229)

[16] V. S. L'vov, E. Podivilov, A. Pomyalov, I. Procaccia, and D. Vandembroucq, Improved shell model of turbulence, Phys. Rev. E (3) 58 (1998), 1811-1822. MR.1637121

[17] A. M. Obukhov, Some general properties of equations describing the dynamics of the atmosphere, Izv. Akad. Nauk SSSR Ser. Fiz. Atmosfer. i Okeana 7 (1971), 695-704.

[18] K. Ohkitani and M. Yamada, Temporal intermittency in the energy cascade process and local Lyapunov analysis in fully-developed model of turbulence, Progr. Theoret. Phys. 81 (1989), 329-341. MR997440 (90j:76065)

[19] R. M. S. Rosa, Asymptotic regularity condition for the strong convergence towards weak limit sets and weak attractors of the 3D Navier-Stokes equations, J. Differential Equations 229 (2006), 257-269. MR2265627 
[20] R. Temam, Navier-Stokes Equations: Theory and numerical analysis, North-Holland, Amsterdam, 1984. MR769654 (86m:76003)

[21] F. Waleffe, On some dyadic models of the Euler equations, Proc. Amer. Math. Soc. 134 (2006), 2913-2922. MR2231615 (2007g:35222)

Department of Mathematics, University of Michigan, Ann Arbor, Michigan 48109

E-mail address: acheskid@umich.edu

Current address: Department of Mathematics, University of Chicago, 5734 S. University Avenue, Chicago, Illinois 60637

E-mail address: acheskid@uchicago.edu 